\title{
Tempo de permanência prolongado na emergência associado à delirium em idosos:
}

\section{revisão sistemática}

\author{
Prolonged length of stay in the emergency department associated with delirium in the elderly: a \\ systematic review
}

Duración prolongada de la estancia en el servicio de urgencias asociada al delirium en los ancianos: una revisión sistemática

\section{Resumo}

O estudo tem como objetivo analisar se o tempo de permanência no departamento de emergência antes da internação hospitalar é associado à delirium em idosos. Trata-se de uma revisão sistemática realizada segundo o guideline Systematic Reviews and Meta-Analysis (PRISMA). A pesquisa foi realizada nas bases de dados PubMed, SCOPUS, Web of Science, CINAHAL e Google Scholar, bem como de uma busca manual nas listas de referências dos artigos selecionados. Após a análise dos critérios de elegibilidade, três estudos foram incluídos e apresentaram como delineamento coorte prospectiva ou retrospectiva e avaliaram a relação entre incidência de delirium e tempo de permanência de pacientes idosos no Departamento de Emergência. Todos os participantes dos estudos tiveram um tempo de exposição ao departamento de emergência maior ou igual a 8 horas antes de serem encaminhados para a internação hospitalar. $\mathrm{O}$ estudo permite concluir que a permanência prolongada de pacientes idosos no Pronto-Socorro ou Departamento de emergência aumentou o risco de desenvolver delirium.

Palavras-chave: Delirium; Idoso; Serviços médicos de emergência.

\section{Abstract}

The study aims to analyze whether the length of stay in the emergency department before hospital admission is associated with delirium in the elderly. This is a systematic review carried out according to the Systematic Reviews and Meta-Analysis (PRISMA) guideline. The search was performed in PubMed, SCOPUS, Web of Science, CINAHAL and Google Scholar databases, in addition to a manual search of the reference lists of the selected articles. After analyzing the eligibility criteria, three studies were included and presented as prospective or retrospective cohort design and evaluated the relationship between incidence of delirium and length of stay of elderly patients in the 
Emergency Department. All study participants had a length of exposure to the emergency department greater than or equal to 8 hours before being referred for hospital admission. The study allows us to conclude that the prolonged stay of elderly patients in the Emergency Room or Emergency Department increases the risk of developing delirium.

Keywords: Delirium; Aged; Emergency medical services.

\section{Resumen}

El estúdio tiene como objetivo analizar si la duración de la estancia en el servicio de urgencias antes del ingreso hospitalario se asocia con el delirio en los ancianos. Se trata de uma revisión sistemática realizada de acuerdo con la guía de Revisiones Sistemáticas y Meta-Análisis (PRISMA). La búsqueda se realizó en las bases de datos PubMed, SCOPUS, Web of Science, CINAHAL y Google Scholar, además de una búsqueda manual en las listas de referencias de los artículos seleccionados. Tras el análisis de los criterios de elegibilidad, se incluyeron tres estudios que se presentaron con un diseño de cohorte prospectivo o retrospectivo y que evaluaron la relación entre la incidencia de delirio y la duración de la estancia de los pacientes ancianos en el Servicio de Urgencias. Todos los participantes en el estudio tuvieron un tiempo de exposición al servicio de urgencias mayor o igual a 8 horas antes de ser remitidos para su ingreso en el hospital. El estúdio permite concluir que la estancia prolongada de los pacientes de edad avanzada en el Servicio de Urgencias o en el Departamento de Emergencias aumenta el riesgo de desarrollar delirios.

Palabras clave: Delirio; Anciano; Servicios médicos de urgencia.

\section{Introdução}

A superlotação dos Pronto-socorros (PS) é um problema mundial que afeta o sistema de saúde e a segurança do paciente (Bittencourt et al., 2020; Morley et al., 2018), resulta do aumento no volume de pacientes e diminuição da capacidade de leitos de internação do hospital (Pierce \& Gormley, 2016) e vem sendo associado ao aumento do tempo de permanência no PS, redução da qualidade da assistência e desfechos desfavoráveis (Ackroyd-Stolarz et al., 2011; Sri-on et al., 2014).

A demanda por atendimento de emergência, mesmo na maioria países desenvolvidos, cresceu consideravelmente, devido, entre outras coisas, ao aumento na expectativa de vida e, consequentemente, predomínio de doenças crônicodegenerativas, muitas vezes exacerbado (Berchet, 2015).

A principal causa dessa superlotação, é a internação no PS ou boarding (Morley et al., 2018; Ackroyd-Stolarz et al., 2011; Schneider et al., 2003) definida como a permanência dos pacientes no PS, por falta de leitos hospitalares, após a decisão de internar o paciente (Coil et al., 2016; Lord et al., 2018; Mckenna et al., 2019; Salehi et al., 2018). Esses, estão vulneráveis porque não recebem os cuidados necessários que receberiam nas enfermarias (Ackroyd-Stolarz et al., 2011; Schneider et al., 2003), e além disso, estudos apontaram sua correlação com a ocorrência de eventos adversos como: atraso na administração de medicamentos (Coil et al., 2016; Lord et al., 2018; Mckenna et al., 2019), aumento da mortalidade (Schneider et al., 2003; Salehi et al., 2018) e redução da qualidade do cuidado (Bouldi et al., 2020).

Estudo retrospectivo conduzido no Canadá identificaram os impactos do boarding no tempo total de internação e maiores tempos "on boading" são vivenciados por pacientes, com idade próxima de 60 anos, que necessitavam de leitos de clínica médica e tecnologias de cuidado mais avançadas devido ao elevado grau de dependência (Salehi et al., 2018).

A dependência e envelhecimento da população mundial são desafiadores no sistema de saúde, uma vez que a população idosa, cada vez mais numerosa, apresenta problemas de saúde mais complexos, que requerem cuidados especializados, constituindo-se os maiores consumidores dos principais serviços de saúde (Oliveira et al., 2016; Sanon et al., 2014), que repercute em maiores custos hospitalares e impactam na economia.

$\mathrm{Na}$ Coreia do Sul, por exemplo, cerca de $15 \%$ da população tem mais de 64 anos e estudo multicêntrico nesse país mostrou que o aumento da idade elevou a demanda por assistência médica e os custos com despesas com saúde em idosos com 65 anos ou mais, aumentaram de 31,6\% em 2010 para 36,8\% em 2015 (Kim et al., 2019).

Outrossim, a idade é um fator que precede o desenvolvimento das doenças crônicas como Alzheimer, cânceres, doenças neurodegenerativas e cardiovasculares, além da fragilidade, imobilidade e delirium que são associados à morbimortalidade, hospitalização e custos no sistema de saúde mundial (Oh et al., 2017; Parant, 1990). 
Delirium é um estado agudo confusional, frequente em idosos, caracterizado por início agudo, nível alterado de consciência, curso flutuante e distúrbios na orientação, memória, atenção, pensamento desorganizado e comportamento (Oh et al., 2017; Tchkonia et al., 2018), pode ser associado à doenças graves (Pinheiro et al., 2020), ou não, e a prevalência no departamento de emergência varia de 7 a 10\%, em idosos (Átilla et al., 2014), porém apenas 16\% a 35\% dos casos são diagnosticados (Elie et al., 2000; Hustey \& Meldon, 2002).

Este estado confusional é, também, um evento adverso, que repercute na qualidade de cuidado (Pinheiro et al., 2020), associado à piores desfechos (Schubert et al., 2018) Pacientes idosos com delirium possuem 1,5 maior risco de óbito (Smith \& Meyfroidt, 2017) e duplicam o tempo de permanência no hospital, o que acarreta no dobro de horas de profissionais de saúde e dobram os custos totais da internação por paciente (Pinheiro et al., 2020).

Portanto, essa revisão tem por objetivo analisar se o tempo de permanência no departamento de emergência antes da internação hospitalar é associado à delirium em idosos.

\section{Metodologia}

Revisão sistemática com análise qualitativa, cadastrado na plataforma OSF e registrado no link https://osf.io/x64yc/

\section{Critérios de elegibilidade}

A estratégia PICOT (população, intervenção, comparação, desfecho e tipo de estudo) foi utilizada para formular a pergunta de pesquisa, deste modo o estudo teve como foco o seguinte questionamento: $\mathrm{O}$ tempo de permanência prolongado no departamento de emergência (DE) antes da internação hospitalar está associado ao aumento da taxa de delirium em idosos? Os estudos foram considerados elegíveis se satisfizessem os seguintes critérios: (1) estudos de coorte ou caso controle; (2) idade maior que 60 anos; (3) internados no departamento de emergência; (4) não apresentavam o diagnóstico de delirium na admissão no DE e (5) dados brutos suficientes foram fornecidos para avaliar a associação entre tempo de permanência no departamento de emergência e incidência de delirium.

Este trabalho foi conduzido segundo o guideline Systematic Reviews and Meta-Analysis (PRISMA) e dispensou submissão ao comitê de ética em pesquisa.

\section{Fontes de informação e estratégia de busca}

Uma pesquisa sistemática nas bases de dados PubMed, SCOPUS, Web of Science, CINAHAL e Google Scholar, além de uma busca manual nas listas de referências dos artigos selecionados. A busca foi realizada em Junho de 2021, sem restrições de idioma e incluiu publicações dos últimos 10 anos, utilizando os seguintes termos: "elderly patient OR older patient"; "Length of Stay in the Emergency Department OR boarding in the Emergency Department OR boarding OR ED boarded OR ED overcrowded OR ED crowded OR ED crowding OR ED overcrowding OR admission delay OR delayed transfer OR delayed admission OR ED Inpatient Boarding" e "Delirium".

\section{Seleção dos estudos}

Três investigadores examinaram independentemente os resultados da pesquisa e identificaram estudos potencialmente relevantes com base em títulos e resumos. Os estudos relevantes foram lidos na íntegra e selecionados de acordo com os critérios de elegibilidade. As discordâncias entre os três revisores foram resolvidas por consenso ou por um quarto revisor.

\section{Processo de coleta de dados}

Quatro investigadores independentes extraíram os dados dos relatórios publicados usando um protocolo predefinido. 
Informações sobre o desenho do estudo, população elegível, distribuição de idade, período de acompanhamento, medidas de desfecho e resultados e seus valores incluído suas estimativas brutas e ajustadas com intervalos de confiança de 95\% (ICs).

\section{Síntese dos resultados e análise}

Para sintetizar os dados dos estudos incluídos foi utilizado um quadro composto pelos autores, título, delineamento do estudo, população estudada, exposição e os desfechos de todos os artigos. Deste modo, foi possível observar idade da população, o tempo médio de exposição ao departamento de emergência e risco de desenvolvimento de delirium.

Para avaliar a qualidade de cada estudo de forma individual foi utilizada a Ferramenta de Avaliação de Qualidade para Coorte Observacional Newcastle-Otawa. A escala Newcastle-Ottawa utiliza um sistema estelar (0 a 9) para avaliar os estudos selecionados em três domínios: seleção da amostra, comparabilidade e resultados. Quanto maior o escore maior a qualidade.

\section{Resultados}

\section{Seleção dos estudos}

A pesquisa inicial localizou 598 artigos, dos quais 6 foram coletados do PubMed, 381 do Web of Science, 9 do SCOPUS, 105 do CINAHL e 97 no Google Scholar. Oito estudos foram potencialmente relevantes e foram analisados na íntegra. Após uma leitura completa, foram excluídos 5 estudos: 01 por delineamento e 06 por desfecho.

Finalmente, 3 estudos preencheram os critérios de elegibilidade (Émond et al., 2018; Émond et al., 2017 e Bo, 2016) e foram incluídos nessa revisão sistemática. Um fluxograma representando o processo de seleção de referências em cada etapa é fornecido na Figura 1. 
Figura 1. Diagrama de fluxo de pesquisa de literatura e processo de triagem.
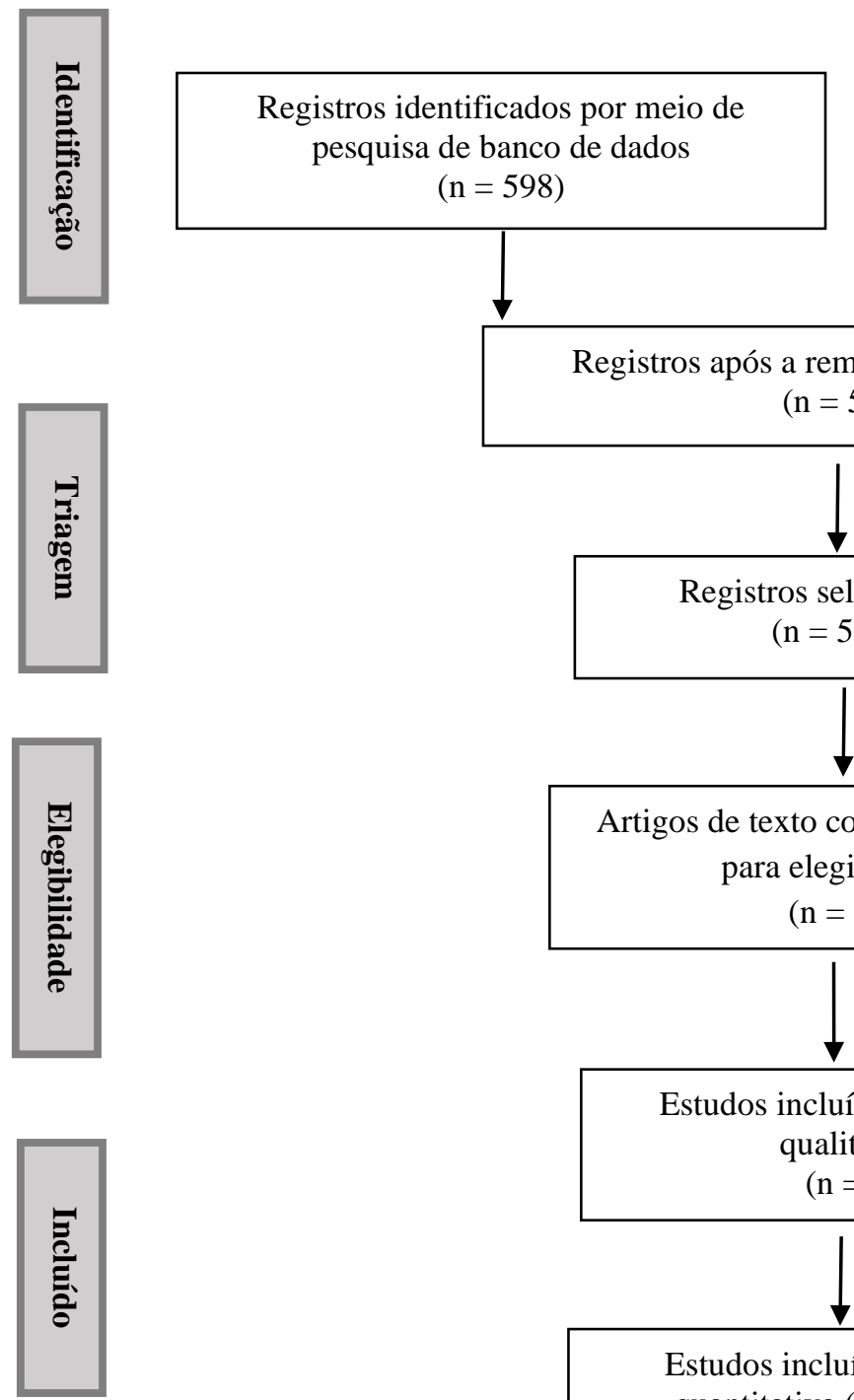

\section{Registros adicionais identificados por} outras fontes $(\mathrm{n}=0)$

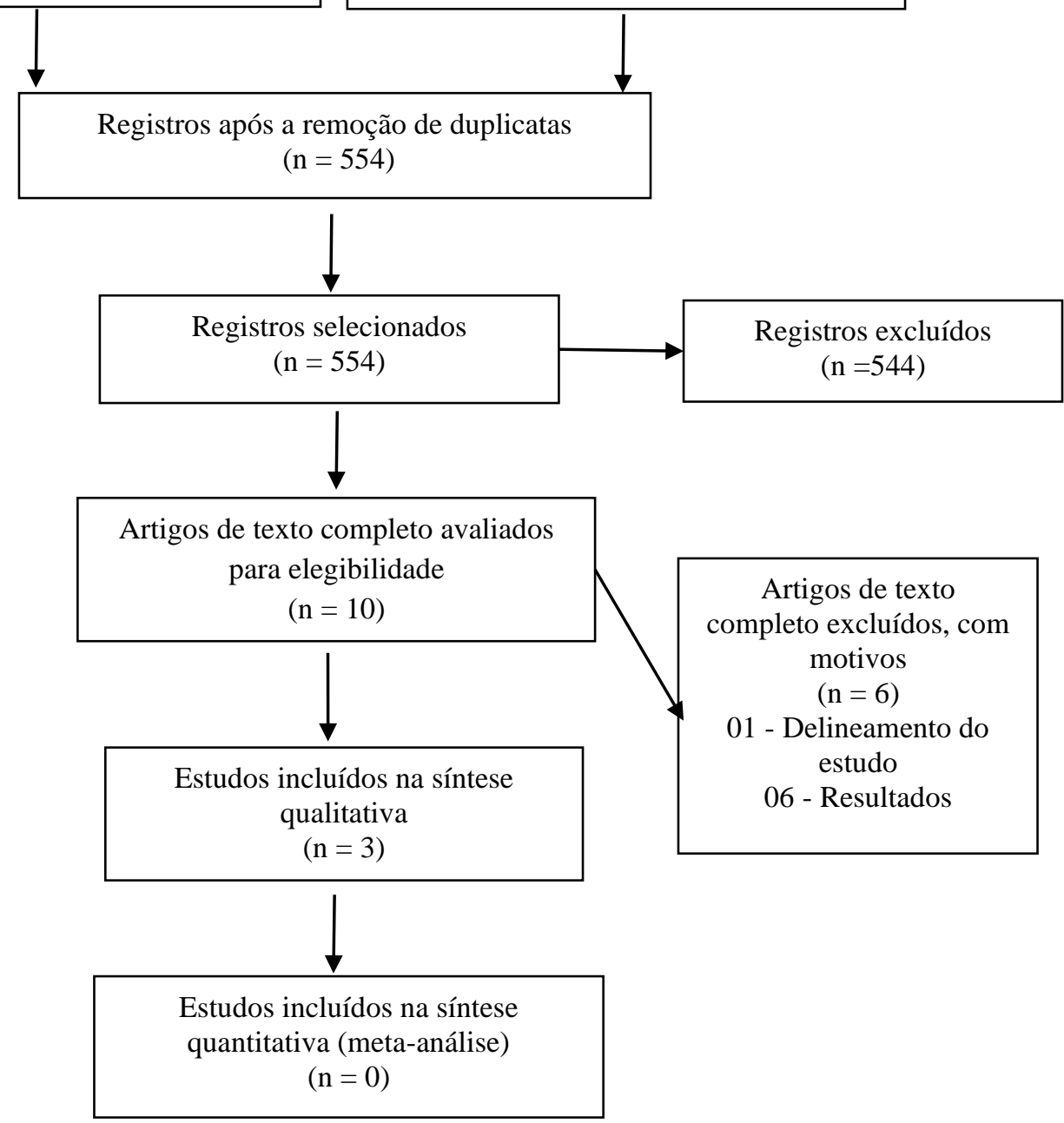

Fonte: Autores.

\section{Qualidade dos estudos}

Os estudos incluídos nesta revisão sistemática destacaram a pergunta de pesquisa, os critérios de elegibilidade e apresentaram baixo risco de viés de seleção. Utilizaram amostras de base populacional e foram ajustados para variáveis de confusão afim de fortalecer a evidência observacional entre o tempo de permanência no departamento de emergência e o delirium em idosos (Tabela 1). 
Tabela 1. Avaliação da qualidade de estudos selecionados usando a escala Newcastle-Ottawa.

\begin{tabular}{|c|c|c|c|c|c|c|c|c|c|}
\hline Estudos de & Seleção & & & & Comparabilidade & Resultado & & & Total \\
\hline Autores & $\begin{array}{l}\text { Representat } \\
\text { ividade da } \\
\text { coorte } \\
\text { exposta }\end{array}$ & $\begin{array}{l}\text { Seleção } \\
\text { da coorte } \\
\text { não } \\
\text { exposta }\end{array}$ & $\begin{array}{l}\text { Verificação } \\
\text { da exposição }\end{array}$ & \begin{tabular}{l}
\multicolumn{3}{l}{ Demonstração } \\
de que o \\
resultado de \\
interesse não \\
estava presente \\
no início do \\
estudo
\end{tabular} & $\begin{array}{ll}\text { Comparabilidade } & \text { de } \\
\text { coortes com base } & \text { no } \\
\text { projeto ou análise } & \end{array}$ & $\begin{array}{l}\text { Avaliação do } \\
\text { resultado }\end{array}$ & $\begin{array}{l}\mathrm{O} \\
\text { acompanhament } \\
\text { o foi longo o } \\
\text { suficiente para } \\
\text { que } \\
\text { resultados } \\
\text { ocorressem }\end{array}$ & $\begin{array}{l}\text { Adequaç } \\
\text { ão de } \\
\text { acompan } \\
\text { hamento } \\
\text { de } \\
\text { coortes }\end{array}$ & \\
\hline $\begin{array}{l}\text { Émond et } \\
\text { al., } 2018\end{array}$ & $*$ & - & $*$ & $*$ & $*$ & $*$ & $*$ & $*$ & 7 \\
\hline $\begin{array}{l}\text { Émond et } \\
\text { al., } 2017\end{array}$ & $*$ & - & $*$ & $*$ & $*$ & $*$ & $*$ & $*$ & 7 \\
\hline $\begin{array}{l}\text { Bo et al., } \\
2016\end{array}$ & $*$ & - & $*$ & $*$ & $*$ & * & $*$ & $*$ & 7 \\
\hline
\end{tabular}

Legenda: * sistema estelar utilizado pela Newcastle-Ottawa. Fonte: Autores.

\section{Características dos estudos}

Os estudos incluídos foram conduzidos no Canadá (Émond et al., 2018 e Émond et al., 2017) e na Itália (Bo et al., 2016). Dois estudos (Émond et al., 2018 e Bo et al., 2016) apresentaram como delineamento uma coorte prospectiva e um (Émond et al., 2017) como coorte retrospectiva. Os participantes, de todos os estudos, foram selecionados de forma consecutiva por meio de admissão em pronto-socorro ou departamento de emergência e tiveram um tempo de exposição ao DE maior ou igual a 8 horas antes de serem encaminhados para internação hospitalar.

Dados de 886 sujeitos foram analisados nessa revisão sistemática, sendo que, a idade deles era acima de 65 anos. A idade avançada e comprometimento cognitivo moderado a grave foram associados ao maior risco de delirium, e quando apresentado aumenta o tempo de internação em aproximadamente uma semana, além de, elevar o perigo de complicações futuras. O tempo de permanência hospitalar para pacientes com delirium foi de 16,8 dias e o tempo de permanência para pacientes sem delirium foi de 10,3. Assim sendo, quanto maior o tempo de internação, maior risco para ocorrência de delirium.

\section{Tempo de permanência hospitalar associado à delirium}

Os três estudos avaliaram a relação entre incidência de delirium e tempo de permanência de pacientes idosos no Departamento de Emergência.

O estudo de Émond, et al., observou que pacientes expostos por no mínimo 12 horas no Departamento de Emergência possuem tempo médio de internação de 16 dias, pacientes sem delirium permaneceram 11,9 dias, enquanto pacientes com delirium permaneceram 8,6 mais dias. Cerca de 20\% dos pacientes desenvolve delirium e metade dos casos ocorre nas primeiras 24 horas após a exposição ao departamento de emergência. O segundo estudo de Émond, realizado em 2018, expõem que após a exposição de 8 horas no Departamento de Emergência 12\% dos 338 pacientes desenvolveram delirium, sendo que o tempo médio de internação hospitalar para os pacientes delirantes foi de 13,1 dias, em contrapartida os pacientes sem delirium permaneceu por 8,7 dias.

Consoante com Émond, o estudo de Bo et al., demostrou que a exposição por mais de 10 horas no Departamento de Emergência foi associada a mais de duas vezes o risco de desenvolver delirium nas 72 horas seguintes. Dessarte, pacientes mais velhos e com comprometimento cognitivo, possuem maior risco de desenvolver delirium, (Bo et al.,2016) outrossim a incidência de delirium, aumenta o tempo de permanência hospitalar e consequentemente aumenta a exposição e risco, (Émond, et al., 2018), decorrentes da internação.

\section{Discussão}

Pacientes idosos expostos ao departamento de emergência por em média 10 horas possuem maior risco de 
desenvolver delirium e consequentemente permanecem por maior tempo internados. Consoante ao resultado obtido nesta revisão, um estudo prospectivo observacional, além de comprovar uma tendência para um maior tempo de internamento na amostra com delirium, relacionou a ocorrência de delirium, 26\%, com maior mortalidade durante o internamento e maior sobrecargas dos profissionais de saúde (Émond et al., 2017).

Um estudo com pacientes idosos e expostos a um tempo de permanência no PS maior ou igual a 8 horas apontou que entre pacientes com dor intensidade $\geq 65$, mensurada pela escala visual analógica (VAS) avaliada de 0 a 100, 26\% experimentaram delirium em comparação com $11 \%$ para pacientes com dor $<65$ ( p <0,01), e mostrou também que pacientes que tiveram dor intensa na primeira entrevista tinha 3,3 (OR: IC 95\%: 1,4-7,9) vezes mais probabilidade de sofrer de delirium. Além disso, nenhuma associação foi observada entre os opióides recebidos durante a internação no pronto-socorro e delirium em análises multivariadas ( $\mathrm{p}=0,63$ ) (Daoust et al., 2020).

Em uma das maiores coorte realizadas recentemente sobre transtornos do espectro cognitivo - delirium e demência em pacientes com 65 anos ou mais hospitalizados, relatou a ocorrência de 24,6\% de delirium em de todas as admissões e maior tempo de permanência para pacientes com delirium, cerca de 14 dias a mais em comparação aos pacientes sem transtornos do espectro cognitivo, ademais idosos com um transtorno do espectro cognitivo apresentaram maior mortalidade no ano após a admissão (40,0\% vs. $26,0 \%)$ e maior mortalidade ou readmissão no ano após a alta (62,4\% vs. 51,5\%) (Reynish et al., 2017).

O estudo de coorte realizado por Fuchs demostrou que os pacientes delirantes eram mais velhos e mais frequentemente admitidos na emergência, em consenso Reynish evidenciou que o aumento da idade está fortemente relacionado a prevalência de delirium, aumentando de 18,4\% em pessoas com 65-69 anos para mais de 50\% em pessoas com 85 anos ou mais (Reynish et al., 2017; Fuchs et al., 2020).

O estudo observacional prospectivo, realizado em 2020, com 745 pacientes, com 65 anos ou mais, demostrou que os pacientes que desenvolveram delirium era mais velhos e permaneciam por mais tempo nas unidades médicas quando comparado ao grupo de pacientes não delirantes. As principais causas de admissão hospitalar desses pacientes foram as doenças do sistema circulatório, $73 \%$, e do sistema nervoso, cerca de $16 \%$, seguidas de doenças do sistema respiratório e doenças infecciosas e parasitárias, ambas com a média de 3\% das admissões (Bergjan et al., 2020).

Destarte, ao analisar os fatores precipitantes de delirium em idosos hospitalizados, é possível destacar a presença de demência, dor e problemas de mobilidade relevantes como o risco de quedas, o risco de úlcera por pressão e imobilidade, além disso a polifarmácia, uso de mais de sete medicamentos, também foi considerada um fator de risco significativo (Bergjan et al., 2020; Maciel et al., 2021).

Outrossim, a realização de cirurgias, também é uma condição predisponente para delirium (Maciel et al., 2021), sendo mais frequente após procedimentos ortopédicos, cardiovasculares, neurológicos e viscerais, o estudo de coorte realizado nos Estados Unidos, observou que a frequência de delirium pós-operatório aumentou de forma significativa com a idade dos pacientes e está associada a um risco aumentado de diagnóstico subsequente de comprometimento cognitivo leve ou demência (Fuchs et al., 2017; Sprung et al., 2017).

\section{Conclusão}

A permanência prolongada no PS aumenta as chances de desenvolver delirium na população idosa. A redução da qualidade da assistência prestada a pacientes internados no PS parece expor o paciente a complicações que prolongam o seu tempo de internação e contribuiu para o aumento de comorbidades.

Diante do exposto, é necessário que sejam realizados mais estudos que busquem responder de forma mais assertiva a essa correlação e que exponham os fatores que podem estar relacionados a esse desfecho e com isso propor estratégias que minimizem a ocorrência do delirium no PS e qualifique o cuidado destinado a população idosa. 


\section{Referências}

Ackroyd-Stolarz, S., Read Guernsey, J., Mackinnon, N. J., \& Kovacs, G. (2011). The association between a prolonged stay in the emergency department and adverse events in older patients admitted to hospital: a retrospective cohort study. BMJ quality \& safety, 20(7), 564-569. https://doi.org/10.1136/bmjqs.2009.034926

Atilla, Ö. D., Sezík, S., Dağar, S., Akkaya, A., \& Aksay, E. (2014). Delirium in older emergency department patients is associated with increased in-hospital mortality. Turk Geriatr Derg, 17(1), 57-62

Berchet, C. (2015). Emergency care services: trends, drivers and interventions to manage demand, OECD Health Working Papers, (83). https://doi.org/10.1787/18152015

Bergjan, M., Zilezinski, M., Schwalbach, T., Franke, C., Erdur, H., Audebert, H. J., \& Hauß, A. (2020). Validation of two nurse-based screening tools for delirium in elderly patients in general medical wards. BMC Nurs, 19(1), 1-9. https://dx.doi.org/10.1186\%2Fs12912-020-00464-4

Bittencourt, R. J., Stevanato, A. M., Bragança, C. T. N. M., Gottems, L. B. D., \& O’Dwyer, G. (2020). Interventions in overcrowding of emergency departments: an overview of systematic reviews. Rev Saude Publica, 54(66). https://doi.org/10.11606/s1518-8787.2020054002342

Bo, M., Bonetto, M., Bottignole, G., Porrino, P., Coppo, E., Tibaldi, M., ... \& Bellelli, G. (2016). Length of Stay in the Emergency Department and Occurrence of Delirium in Older Medical Patients. Journal of the American Geriatrics Society, 64(5), 1114-1119. https://doi.org/10.1111/jgs.14103

Boudi, Z., Lauque, D., Alsabri, M., Östlundh, L., Oneyji, C., Khalemsky, A., .. \& Bellou, A. (2020). Association between boarding in the emergency department and in-hospital mortality: A systematic review. PLoS One, 15(4), e0231253. https://doi.org/10.1371/journal.pone.0231253

Coil, C. J., Flood, J. D., Belyeu, B. M., Young, P., Kaji, A.H., \& Lewis, R.J. (2016). The Effect of Emergency Department Boarding on Order Completion. Ann Emerg Med, 67 (6), 730-736. https://doi.org/10.1016/j.annemergmed.2015.09.018

Daoust, R., Paquet, J., Boucher, V., Pelletier, M., Gouin, É., \& Émond, M. (2020). Relationship Between Pain, Opioid Treatment, and Delirium in Older Emergency Department Patients. Academic emergency medicine: official journal of the Society for Academic Emergency Medicine, 27(8), 708-16. https://doi.org/10.1111/acem.14033

Elie, M., Rousseau, F., Cole, M., Primeau, F., McCusker, J., \& Bellavance, F. (2000). Prevalence and detection of delirium in elderly emergency department patients. CMAJ, 163(8), 977-81. http://www.ncbi.nlm.nih.gov/pubmed/11068569

Émond, M., Boucher, V., Carmichael, P. H., Voyer, P., Pelletier, M., Gouin, É., ... \& Lee, J. (2018). Incidence of delirium in the Canadian emergency department and its consequences on hospital length of stay: A prospective observational multicentre cohort study. BMJ Open, 8(3), e018190. https://doi.org/10.1136/bmjopen-2017-018190

Émond, M., Grenier, D., Morin, J., Eagles, D., Boucher, V., Le Sage, N., ... \& Lee, J. S. (2017). Emergency department stay associated delirium in older patients. Can Geriatr J, 20(1), 10-14. https://doi.org/10.5770/cgj.20.246

Fuchs, S., Bode, L., Ernst, J., Marquetand, J., Känel, V. R, \& Böttger S. (2020). Delirium in elderly patients: Prospective prevalence across hospital services. Gen Hosp Psychiatry, 67, 19-25. https://doi.org/10.1016/j.genhosppsych.2020.08.010

Hustey, F. M., \& Meldon, S. W. (2002). The prevalence and documentation of impaired mental status in elderly emergency department patients. Annals of Emergency Medicine, 39(3), 248-253. https://doi.org/10.1067/mem.2002.122057

Kim, K., Lee, D. H., Yune, H.Y., Wee, J. H., Kim, D.H., Kim, E. C., ... Choi, S. P. (2019). Identifying Potentially Avoidable Emergency Department Visits of Long-Term Care Hospital Residents in Korea: A Multicenter Retrospective Cohort Study. BioMed Research International, 2019, 1-7. https://doi.org/10.1155/2019/7041607

Lord, K., Parwani, V., Ulrich, A., Finn, E.B., Rothenberg, C., ... \& Rosenberg, A. (2018). Emergency department boarding and adverse hospitalization outcomes among patients admitted to a general medical service. Am J Emerg Med, 36(7), 1246-1248. https://doi.org/10.1016/j.ajem.2018.03.043

Maciel, M. C., Niwa, L. M. S., Ciosak, S. I., \& Najas, M. S. (2021). Fatores Precipitantes de Delirium em Pacientes Idosos Hospitalizados. Rev Divulg Científica Sena Aires, 10(1), 117-26. https://doi.org/10.36239/revisa.v10.n1.p117a126

McKenna, P., Heslin, S. M., Viccellio, P., Mallon, W. K., Hernandez, C., \& Morley, E .J. (2019). Emergency department and hospital crowding: causes, consequences, and cures. Clin Exp Emerg Med, 6(3), 189-95. https://doi.org/10.15441/ceem.18.022

Morley. C., Unwin, M., Peterson, G. M., Stankovich, J., \& Kinsman, L. (2018). Emergency department crowding: A systematic review of causes, consequences and solutions. PLoS One, 13(8), e0203316. https://dx.plos.org/10.1371/journal.pone.0203316

Oh, E. S., Fong, T. G., Hshieh, T. T., \& Inouye, S. K. (2017). Delirium in Older Persons. JAMA, 318(12), 1161. https://doi.org/10.1001/jama.2017.12067

Oliveira, M. R., Veras, R.P., Cordeiro, H. A., \& Pasinato, M.T. (2016). A mudança de modelo assistencial de cuidado ao idoso na Saúde Suplementar: identificação de seus pontos-chave e obstáculos para implementação. Physis Rev Saúde Coletiva, 26(4), 1383-94. https://doi.org/10.1590/S010373312016000400016

Parant, A. (1990). Les perspectives demographiques mondiales [World population prospects]. Futuribles (Paris, France: 1981), (141), 49-78. http://www.ncbi.nlm.nih.gov/pubmed/12283219

Pierce, B. A., \& Gormley, D. (2016). Are Split Flow and Provider in Triage Models in the Emergency Department Effective in Reducing Discharge Length of Stay? J Emerg Nurs, 42(6), 487-491. http://dx.doi.org/10.1016/j.jen.2016.01.005 
Research, Society and Development, v. 11, n. 1, e32511124966, 2022

(CC BY 4.0) | ISSN 2525-3409 | DOI: http://dx.doi.org/10.33448/rsd-v11i1.24966

Pinheiro, F. G. M. S., Santos, S. E., Barreto, Í. D. C., Weiss, C., Vaez, A. C., Oliveira, J. C., ... \& Silva, F. A. (2020). Mortality Predictors and Associated Factors in Patients in the Intensive Care Unit: A Cross-Sectional Study. Crit Care Res Pract, 2020, 1-6. https://doi.org/10.1155/2020/1483827

Quaresma, F., Duarte, A. M. L., Reis-Pina, P. S. S., Neto, I. M. M. A. G., \& Almeida, J. (2020). Delirium numa enfermaria de Medicina Interna - impacto na prática clínica. Rev Med, 99(4), 357-365. https://doi.org/10.11606/issn.1679-9836.v99i4p357-365

Reynish, E. L., Hapca, S. M., Souza, N., Cvoro, V., Donnan, P.T., \& Guthrie, B. (2017). Epidemiology and outcomes of people with dementia, delirium, and unspecified cognitive impairment in the general hospital: prospective cohort study of 10,014 admissions. BMC Med, 15(1), 140. https://doi.org/10.1186/s12916-017-0899-0

Salehi, L., Phalpher, P., Valani, R., Meaney, C., Amin, Q., Ferrari, K., \& Mercuri, M. (2018). Emergency department boarding: a descriptive analysis and measurement of impact on outcomes. CJEM, 20(6), 929-37. http://doi.org/10.1017/cem.2018.18

Sanon, M., Baumlin, K. M., Kaplan, S. S, \& Grudzen, C. R. (2014). Care and respect for elders in emergencies program: A preliminary report of a volunteer approach to enhance care in the emergency department. J Am Geriatr Soc, 62(2), 365-70. https://doi.org/10.1111/jgs.12646

Schneider, S. M., Gallery, M. E., Schafermeyer, R., \& Zwemer, F. L. (2003). Emergency department crowding: A point in time. Ann Emerg Med, 42(2), 167172. https://doi.org/10.1067/mem.2003.258

Schubert, M., Schürch, R., Boettger, S., Nuñez, G. D., Schwarz, U., Bettex, D., ... \& Rudiger, A. (2018). A hospital-wide evaluation of delirium prevalence and outcomes in acute care patients - A cohort study. BMC Health Serv Res, 18(1), 550. https://doi.org/10.1186/s12913-018-3345-x

Smith, M., \& Meyfroidt, G. (2017). Critical illness: the brain is always in the line of fire. Intensive Care Medicine, 43(6), 870-873. https://doi.org/10.1007/s00134-017-4791-3

Sprung, J., Roberts, R. O., Weingarten, T. N., Calvacante, A. N., Knopman, D. S., Petersen, R. C., ... \& Warner, D. O. (2017). Postoperative delirium in elderly patients is associated with subsequent cognitive impairment. Br J Anaesth, 119(2), 316-323. https://doi.org/10.1093/bja/aex130

Sri-on, J., Chang, Y., Curley, D. P., Jr, C. A. C., Weissman, J. S., Singer, S. J., \& Liu, S. W. (2014). American Journal of Emergency Medicine Boarding is associated with higher rates of medication delays and adverse events but fewer laboratory-related delays. Am J Emerg Med, 32(9), 1033-1036. http://dx.doi.org/10.1016/j.ajem.2014.06.001

Tchkonia, T., \& Kirkland, J. L. (2018). Aging Cell Senescence, and Chronic Disease: Emerging Therapeutic Strategies. JAMA, 320(13), 1319-1320. https://doi.org/10.1001/jama.2018.12440 